\title{
Debate Estruturado: Uma Estratégia Pedagógica para Ensino e Aprendizagem de Valores Humanos em Interação Humano-Computador
}

\author{
Lesandro Ponciano \\ Pontifícia Universidade Católica de Minas Gerais \\ Belo Horizonte, Minas Gerais, Brasil \\ lesandrop@pucminas.br
}

\begin{abstract}
Interactive systems are increasingly present in people's daily lives, generating new demands for interface and interaction. Examples of demands are those derived from human values, such as: gender identity, privacy, and the need for systems to provide explanations to the people who use them. Therefore, to be successful, professionals working in the area of HumanComputer Interaction (HCI) need to be increasingly sensitive to these values. Unfortunately, there are still few pedagogical resources to help HCI students develop such sensitivity. Seeking to contribute to the solution of this problem, in this work, it is proposed and analyzed the use of the pedagogical strategy denominated Structured Debate. Based on the pedagogies of active learning and collaborative learning, Structured Debate follows three phases: 1) pre-debate phase, which consists of preparing for the debate by reading reference texts; 2 ) execution of the debate in the classroom through a structure of questions, answers, considerations and movements in the room; and, 3) post-debate phase, when the effects of the debate are observed and contextualized in new contents of the discipline. We discuss experiences of the use of the strategy in five classes of the discipline HCI in two bachelor undergraduate courses: Information Systems and Software Engineering. The results suggest that Structured Debate stimulates and engages students with HCI topics about human values.
\end{abstract}

\section{Author Keywords}

Debate, Education, Human Values, Educational Resources

\section{ACM Classification Keywords}

H.5.m : Information interfaces and presentation (e.g., HCI): HCI Education.

\section{INTRODUÇÃO}

À medida que os sistemas computacionais se tornam mais presentes no cotidiano das pessoas, os profissionais que atuam

Permission to make digital or hard copies of all or part of this work for personal or classroom use is granted without fee provided that copies are not made or distributed for profit or commercial advantage and that copies bear this notice and the full citation on the first page. Copyrights for components of this work owned by others than the author(s) must be honored. Abstracting with credit is permitted. To copy otherwise, or republish, to post on servers or to redistribute to lists, requires prior specific permission and/or a fee. Copyright 2018 SBC.

IHC 2018, Anais Estendidos do XVII Simpósio Brasileiro sobre Fatores Humanos em Sistemas Computacionais

Outubro 22-26, 2018, Belém, Brasil

Workshop sobre Educação em IHC (WEIHC) com Interação Humano-Computador (IHC) precisam ser mais sensíveis aos valores humanos que se encontram em questão no contexto dos sistemas e aos fenômenos associados aos seus usos, pois ambos podem despertar novas demandas em interface e interação $[4,10,15,19]$. Esse é o caso, por exemplo, das questões de gênero e de identidade de gênero [7, 17], dos diferentes perfis de preocupação com privacidade $[14,20]$ e do aprendizado de tecnologia e por meio da tecnologia [6,9].

O conteúdo abordado na disciplina IHC se estende desde os arcabouços teóricos que guiam o projeto, implementação e avaliação de sistemas interativos até a compreensão dos mais diversos fenômenos associados aos seus usos [4, 16]. O estudante deve desenvolver competências e habilidades para lidar com situações diversas, desde aquelas de conhecimento comum, às quais se aplica um racionalismo técnico, até aquelas mais singulares, provocadas por novos contextos e que requerem uma reflexão em ação. A aprendizagem não se atém apenas às questões técnicas, envolve a capacidade de sentir $e$ agir diante das demandas provocadas pelos novos contextos.

A definição de estratégias pedagógicas que ajudem o estudante a desenvolver a sensibilidade para compreender e lidar com situações de projeto envolvendo valores humanos ainda é um desafio a ser superado. Buscando contribuir com a análise de novos recursos pedagógicos que podem ser empregados no ensino de valores humanos em IHC, neste trabalho, propõe-se e analisa-se a estratégia de Debate Estruturado. A estratégia tem fundamentos nas pedagogias da aprendizagem ativa e aprendizagem colaborativa [2,21]. Os objetivos deste estudo são apresentar a estratégia e contextualizar o seu uso reportando experiências obtidas ao aplicá-la em turmas da disciplina IHC.

O Debate Estruturado possui uma fase de preparação (prédebate), uma fase de execução (dinâmica em sala) e uma fase de análise (pós-debate). Cada debate tem como objeto de análise um valor humano em IHC, que é definido como o tópico do debate. A fase de preparação consiste nos estudantes lerem artigos científicos e escreverem análises tratando do tópico. A fase de execução é guiada por uma sequência estruturante de perguntas, respostas, percepções e movimentações em sala de aula. Por fim, a fase pós-debate consiste na coleta de percepções, observação de comportamentos e instanciamento das discussões nas aulas subsequentes ao debate. 
Conduziu-se o Debate Estruturado em 9 estudos de caso, com 5 turmas, sendo 2 turmas do curso Bacharelado em Engenharia de Software e 3 turmas do curso Bacharelado em Sistemas de Informação. Os tópicos dos debates incluem identidade de gênero, privacidade em sistemas ubíquos, aprendizado por meio de tecnologia e a influência de robôs nas decisões humanas. Entre os resultados, observa-se que a estratégia é eficaz em canalizar, para os tópicos-chave da disciplina, a disposição natural dos estudantes em discutir, dar opiniões e analisar perspectivas. O debate desenvolve, no estudante, sensibilidade aos pontos prós e contras de diversas situações de projeto de interface e interação. Ao partir da leitura de artigos, a estratégia aglutina diversos conteúdos estudados na disciplina, como fundamentos teóricos, diretrizes de projeto e avaliação de IHC.

\section{BASE TEÓRICA E TRABALHOS RELACIONADOS}

Nesta seção, primeiro discute-se a aprendizagem ativa e colaborativa que são os fundamentos teóricos da estratégia proposta. Após isso, apresenta-se trabalhos relacionados ao ensino de IHC e ao uso do debate como estratégia pedagógica.

\section{Aprendizagem Ativa e Colaborativa}

A aprendizagem ativa é um método pedagógico que requer que os estudantes sejam envolvidos em atividades de aprendizagem que sejam significativas e que os levem a pensarem sobre o que eles estão fazendo e por qual razão [2,21]. Nesse sentido, a aprendizagem ativa se contrasta com as tradicionais aulas expositivas nas quais o estudante recebe, passivamente, as informações do professor. Os elementos principais da aprendizagem ativa são o engajamento e a atividade do estudante no processo de aprendizagem. A aprendizagem colaborativa, por sua vez, requer que os estudantes trabalhem juntos em grupos a fim de interagirem entre si para atingirem um objetivo. $\mathrm{O}$ elemento principal da aprendizagem colaborativa é a ênfase nas interações entre os estudantes, em vez de o estudante aprender sozinho em uma atividade individual [21].

O debate de tópicos relevantes ao conteúdo da disciplina remete à aprendizagem ativa quando o estudante é engajado ativamente na discussão questionando e respondendo questionamentos. Têm-se elementos de aprendizagem colaborativa quando os estudantes juntos precisam definir as questões, perceber perspectivas, apresentar respostas e justificar suas escolhas na dinâmica do debate em sala de aula.

\section{Trabalhos Relacionados ao Debate e Ensino de IHC}

Estratégias de debate em sala já são empregadas como recurso pedagógico em disciplinas de diversas áreas do conhecimento, incluindo Sociologia [11, 12], Medicina [5] e Bioética [13]. Nessas disciplinas, a estratégia tem servido a diversos propósitos, como: ajudar o estudante a desenvolver uma sensibilidade para questões éticas, desenvolver a habilidade de falar em público e ser capaz de pensar de forma crítica sobre os tópicos que norteiam o conteúdo da disciplina. O uso da estratégia de debate como recurso pedagógico no contexto da disciplina IHC ainda é algo pouco mencionado na literatura.

Pesquisadores têm buscado o emprego da aprendizagem ativa como meio para ensinar desenvolvimento de software às novas gerações de estudantes $[3,8,18]$. Em disciplinas de IHC, três tipos de estratégias têm sido destacadas [16], são elas: levar os estudantes a revisarem e discutirem histórias que descrevem situações de projeto (case history reviews); realizar estudos controlados nos quais os estudantes são levados a resolverem problemas práticos (problem solving cases); envolver os estudantes em situações em que eles precisam tomar decisões buscando consenso e justificando suas escolhas (decision-making cases). Apesar desses esforços, ainda mostram-se necessárias estratégias que levem os estudantes a pensarem de forma crítica o projeto, desenvolvimento e avaliação de sistemas interativos que requerem cada vez mais sensibilidade para lidar com os valores humanos a eles associados [4, 16, 19].

\section{ESTRATÉGIA DE DEBATE ESTRUTURADO}

O Debate Estruturado parte de um tópico que envolve valores humanos em interface e/ou interação em sistemas interativos. Exemplos de tópicos são privacidade em redes sociais e socialização de recém-chegados em comunidades on-line. A discussão do tópico requer que o estudante se prepare e tal preparação envolve a leitura de artigos de referência, que são textos que servem de base comum para o debate. Uma vez colocados o tópico e os artigos, o debate é estruturado em três fases: pré-debate, execução e pós-debate. Uma visão geral dessas fases é apresentada na Figura 1 e elas são descritas nos próximos parágrafos.

\section{Fase Pré-Debate.}

Trata-se da preparação individual. Ela consiste em cada estudante ler os artigos científicos (geralmente 2) relacionados ao tópico e escrever um documento de 1 página contendo 3 partes: a) análise do artigo 1 ; b) análise do artigo 2; e, c) análise inter-relacionando os artigos 1 e 2 e apresentando as percepções gerais do estudante. Essa fase visa desenvolver no estudante as competências básicas para participar do debate, que são: dominar os principais conceitos associados ao tópico, desenvolver uma opinião acerca do tópico, e ser capaz de levantar questões e fazer análises críticas sobre o tópico. O professor verifica nas análises escritas se as competências foram desenvolvidas. Em caso positivo, o estudante está preparado e participa da fase de execução do debate. Os estudantes que não fizeram recebem feedback individual do professor e podem apenas assistir a fase de execução do debate.

\section{Fase de Execução do Debate}

No dia do debate, no início da aula, os estudantes são recordados sobre: 1) o tópico que será debatido e a importância de não fugir do tópico; 2) as etapas do debate (indicadas na fase de execução na Figura 1);3) a necessidade de articular uma fala que seja clara e de evitar insistirem sempre em ideias já discutidas; 4) respeitarem as medições de tempo e não emitirem opiniões ofensivas aos colegas e/ou aos direitos humanos; e, 5) usarem bem o tempo disponível para as respostas.

A sala é organizada de modo a favorecer o diálogo entre os estudantes. A Figura 2 exemplifica a organização usada. No estilo do Parlamento Britânico, os estudantes são organizados em dois lados (A e B), com aproximadamente o mesmo número de membros. A existência de dois lados tem como principal motivação a alternância das falas entre o lado A e o lado B e a busca por pontos e contrapontos na discussão do tópico. Os estudantes podem se movimentar durante debate, mas 


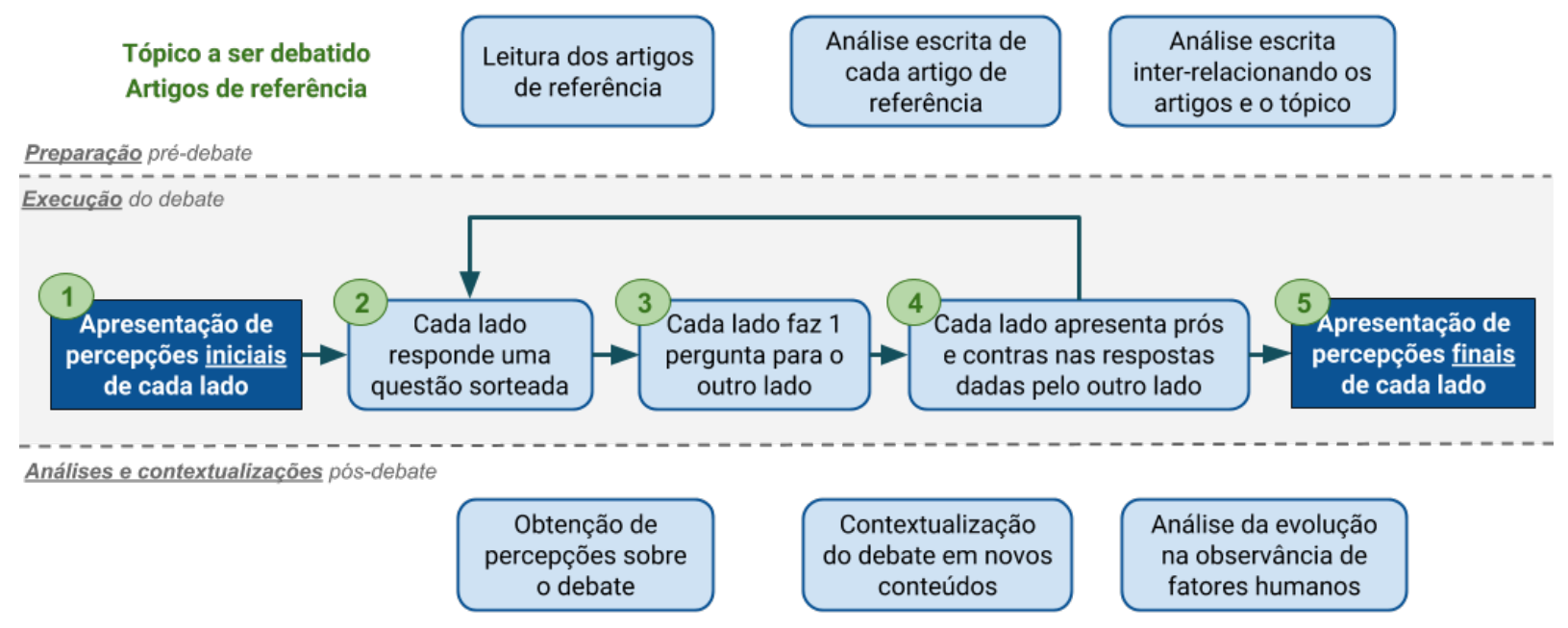

Figura 1. Etapas estruturantes do debate.

os movimentos têm significados. Se o estudante levanta-se e senta-se logo em seguida, ele está manifestando concordância com quem está falando; independente se é do seu lado ou do outro lado. Se o estudante levanta-se e assume uma cadeira no outro lado, ele demonstra que tende a concordar mais com as percepções colocadas por esse lado do que com as colocadas pelo lado em que se encontrava.

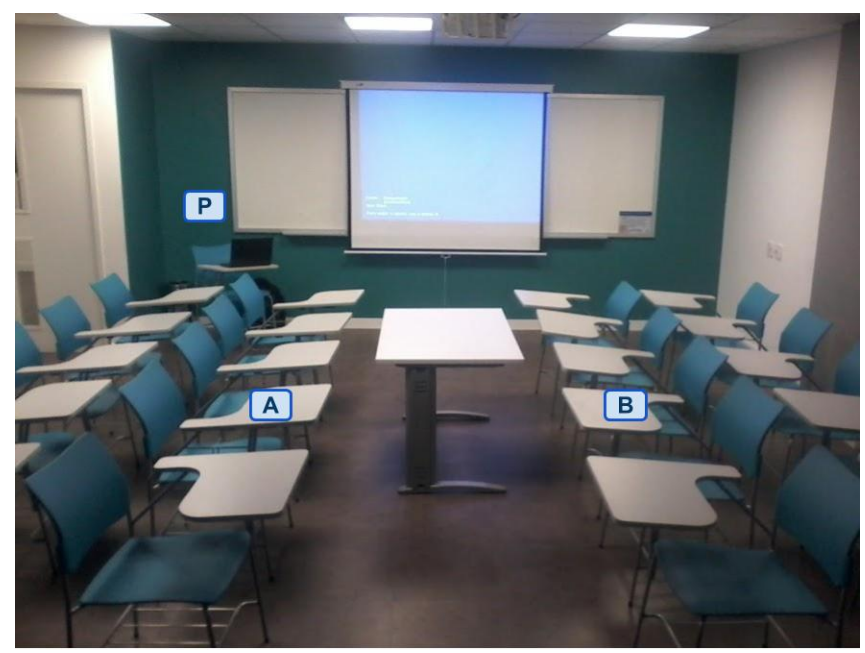

Figura 2. Exemplo de organização da sala em dia de debate. A figura destaca o posicionamento dos lados (A e B) e o posicionamento do professor (P) que indica as atividades do debate projetando-as no datashow.

A fase de execução do debate tem 5 etapas (Figura 1). O debate começa com cada lado apresentando os pontos de vista iniciais sobre o tópico a partir dos artigos lidos. Após isso, na segunda etapa, o professor sorteia questões elaboradas por ele sobre o tópico. As questões do professor são previamente elaboradas, com base nos artigos de referência e nas análises que os estudantes escreveram. Sorteia-se uma questão para o lado A, que a responde. Em seguida, faz-se o mesmo para o lado B. $\mathrm{Na}$ terceira etapa, cada lado pode fazer uma pergunta para o outro lado. Na etapa quatro, cada lado apresenta suas percepções sobre as respostas do outro lado, destacando os pontos positivos e os pontos negativos dessas respostas e eventuais aspectos que deveriam ter sido considerados e que não foram. As etapas 2, 3 e 4 podem ser repetidas. Por fim, o debate é concluído com cada lado apresentando suas percepções finais.

Ao longo do debate, o professor exibe aos estudantes slides que apresentam a etapa atual do debate, quem está com a fala e por quanto tempo. Exemplo de slide usado neste guia é apresentado na Figura 3. Perguntas e respostas devem ser feitas dentro de um tempo máximo acordado previamente com os estudantes. Todas as etapas são cronometradas.

\section{Percepção do Lado A sobre as Respostas do Lado B}

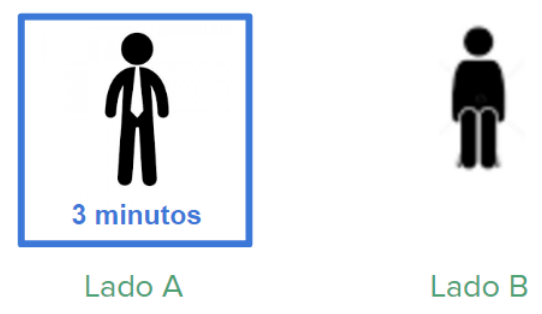

Figura 3. Exemplo de slide guiando as atividades que compõem a fase de execução do debate. Esse exemplo indica que o Labo A tem 3 minutos para comentar sobre as respostas do labo $B$.

\section{Fase Pós-Debate}

A fase pós-debate consiste na investigação, observação e registro dos resultados do debate. Na aula seguinte ao debate, os estudantes são questionados sobre suas percepções acerca do debate. As discussões ocorridas no debate podem ser contextualizadas em conteúdos lecionados em aulas subsequentes ao debate, buscando facilitar a compreensão dos novos conteúdos.

\section{RELATO DE EXPERIÊNCIAS EM 9 ESTUDOS DE CASO Método}

Este trabalho é um relato de experiências. Os debates foram conduzidos em sala com o propósito de ensino de valore huma- 
nos. Após observar-se a boa aceitação e feedback dos alunos, a experiência foi estruturada a partir de anotações diárias do professor para ser reportada. Portanto, segue-se uma abordagem naturalística e qualitativa [1]. Observação naturalística por não envolver manipulação de variáveis, nem tratamento experimental; os debates foram conduzidos e observados no seu acontecer natural. Qualitativo por focar no relatado do que se observa e não na contagem ou medição. Cada debate conduzido em uma turma é tratado como um estudo de caso.

\section{Contexto dos Estudos de Caso}

Os estudos de caso foram conduzidos em três campi da PUC Minas. Foram nove debates, envolvendo 5 turmas de IHC dos cursos Bacharelado em Engenharia de Software (ES) e Bacharelado em Sistemas de Informação (SI). Ambos os cursos são noturnos, possuem ao todo 8 períodos e têm IHC como disciplina obrigatória, com carga horária de 68 horas-aula.

No curso de ES, a disciplina IHC encontra-se alocada no quarto período. O debate foi conduzido em 2 turmas, sendo uma turma no segundo semestre de 2017 (2017-II) e outra turma no primeiro semestre de 2018 (2018-I). A primeira era composta de 11 estudantes e a segunda de 12 estudantes. No curso SI, a disciplina IHC está alocada no quinto período. O debate foi conduzido em 3 turmas, sendo uma turma em 2017II e outras duas turmas em 2018-I. A turma de 2017-II era composta por 18 estudantes. As duas turmas de 2018-I, por sua vez, eram compostas por 26 e 29 estudantes.

A Tabela 1 apresenta os tópicos debatidos, os artigos de referência utilizados e as turmas e semestres nos quais os debates ocorreram. Os tópicos e artigos foram escolhidos de modo a engajar os estudantes em discussões atuais. Eles remetem a valores humanos, decisões de projeto, tipos de interface, paradigmas de interação e avaliação de sistemas interativos.

A seguir são apresentados exemplos de questões criadas e sorteadas pelo professor nos tópicos debatidos:

- "Pode-se dizer que a interface do sistema é sempre neutra e que não ocasiona opressão de gênero?"

- "Se todos os usuários de um sistema reportam que não se preocupam com a privacidade (são unconcerned users), então o designer do sistema é livre para tratar privacidade da forma como melhor lhe convier na interface e na interação?"

- "Na medida em que os sistemas tratam as individualidades dos estudantes e fomentam uma direção personalizada de aprendizado para cada um deles, eles não reduzem a socialização e a cooperação entre os estudantes?"

- "Sistemas computacionais podem determinar as opiniões e ações das pessoas por meio da interface e interação providas, em que medida as pessoas estão sendo controladas atualmente e quais os riscos?"

O tópico do debate e os dois artigos de referência foram informados aos estudantes com pelo menos 45 dias de antecedência ao dia da entrega da análise escrita. A disponibilização dessas informações, a entrega da análise escrita pelo estudante e o fornecimento de feedback pelo professor ocorreram via Sistema Acadêmico. $\mathrm{O}$ debate em sala ocorrem na primeira ou segunda aula após a entrega da análise escrita. Cada debate teve duração de 1 hora e 20 minutos. Eles contaram entre 3 e 4,5 pontos do total de 100 pontos da disciplina. Essa pontuação é por debate e inclui a análise escrita e a presença no debate. Os estudantes foram pontuados pela completude em relação ao solicitado e pela participação, mas não pelo opinião que manifestaram no debate. Conteúdos específicos dos artigos de referência não foram cobrados em prova.

\section{Resultados}

Esta seção apresenta as principais experiências e resultados de cada fase do debate no conjunto dos estudos de caso.

Fase pré-debate. Os estudantes demonstram preocupação em fazer uma análise escrita que os habilitem para o debate. Durante essa fase, dúvidas comuns reportadas são sobre a não compreensão de algum método empregado no artigo ou na limitação de 1 página para escrever a análise. Houve também problemas com idioma inglês, pois os dois artigos de referência do primeiro tópico de 2017-II estão escritos em inglês. A partir dessa ocorrência, nos debates seguintes, passou-se a utilizar artigos de referência escritos em português. Em todos os debates realizados, na análises escritas, os estudantes demonstraram compreensão dos artigos e capacidade de discutir o tópico. Entre a data de entrega da análise escrita e a data do debate, os estudantes criam expectativas e vontade de participar. Um estudante que já estava faltando há 3 aulas, compareceu ao debate e, no início da aula, ao ser questionado sobre as faltas anteriores, ele respondeu "Eu estava atarefado com trabalhos, mas hoje eu vim por que hoje é dia de debate" (E2). Isso ocorreu no dia do segundo debate de 2018-I.

Fase de execução do debate. Os estudantes tendem a começar o debate mais tímidos e receosos sobre o que falar; isso é mais forte no primeiro debate do semestre letivo, quando é a primeira vez que os estudantes participam. Como esperado, as percepções iniciais apresentadas pelos estudantes são mais restritas ao que foi lido nos artigos de referência. As perguntas sorteadas logo no início do debate fazem com que a participação intensifique. Ocorre, durante o debate, dos estudantes falarem de filmes, séries, "memes" e notícias associadas ao tópico de modo a reforçarem suas posições. Por exemplo, no debate sobre gênero, falou-se sobre notícias de vagões exclusivos para mulheres no metrô do Rio de Janeiro, e, no debate sobre privacidade, falou-se sobre episódios da série Black Mirror. No debate sobre robôs sociais, falou-se sobre fake news na eleição presidencial americana de 2016. Há o risco que o debate fuja para tópicos fora da IHC. Porém, na prática, o que se observa é que essas relações e analogias são importantes ao raciocínio que os estudantes tentam construir. Além disso, os limites de tempo para resposta e a alternância de perguntas incluindo perguntas do professor fazem com que o debate retorne ao tópico de IHC. Sempre que há uma pergunta sorteada, retorna-se ao foco da discussão.

As perguntas feitas de um lado para outro e a análise das percepções acerca das respostas originam pontos e contrapontos. Os estudantes tendem a trazer para o debate os pontos que julgaram mais complexos ou os mais polêmicos que encontraram quando leram os artigos ou quando ouviram as respostas dos colegas. Mudanças de participantes de lado são pouco 


\begin{tabular}{l|l|l|l}
\hline Semestre & Tópico do debate & Artigos de ref. & Estudos de caso (turmas) \\
\hline \multirow{2}{*}{$2017-$ II } & Perfis de preocupação com privacidade em sistemas ubíquos & {$[14]$ e $[20]$} & 1 de ES \\
\cline { 2 - 4 } & Questões gênero e de identidade de gênero em interface e interação & {$[17]$ e [7] } & 1 de ES e 1 de SI \\
\hline \multirow{2}{*}{$2018-\mathrm{I}$} & Dificuldades de se aprender tecnologia e via tecnologia & {$[6]$ e [9] } & 1 de ES e 2 de SI \\
\cline { 2 - 4 } & Riscos, benefícios e controles na interação com robôs sociais & {$[22]$ e [23] } & 1 de ES e 2 de SI \\
\hline
\end{tabular}

Tabela 1. Organização geral dos quatro tópicos e artigos de referência abordados no total de 9 estudos de caso.

frequentes, ocorreram apenas em dois debates, sendo um sobre questões de gênero e outro sobre privacidade em sistemas ubíquos. Os estudantes frequentemente se levantam e sentam em seguida demonstrando concordância com o que está sendo dito. Quando o debate é muito acalorado, eles tendem a se esquecer dessas regras de movimentação e começam a falar em vez de se movimentar e, então, precisam ser recordados para que o debate não seja tumultuado com falas simultâneas.

Fase pós-debate. Observa-se mudança de percepção dos estudantes acerca do quão complexo e polêmico pode ser a discussão de um tópico associado a um valor humano em sistemas interativos. Em relação ao debate sobre aprendizado via tecnologia e por meio de tecnologia, um estudante disse: "Eu sinceramente achei que esse debate seria sem graça, mas ficou intenso" (E1). Também se observa que os estudantes se engajam com os tópicos a ponto de valorizar a aula do debate e a sugerir artigos para o próximo debate. Por exemplo, no período de definição dos artigos de referência para o segundo debate do semestre, um estudante enviou mensagens sugerindo um artigo que ele encontrou e que considerou relevante: “(...) Caso julgue o conteúdo interessante, estou compartilhando para a inclusão como leitura sugerida para a preparação do debate" (E3). Observa-se que após o debate o estudante se sente mais à vontade para falar durante as aulas. A recordação do debate pode superar um semestre. Em um dia de debate, o estudante E7, que cursou a disciplina no semestre anterior, passou em frente a sala e notou a organização das cadeiras e questionou "Hoje tem debate de IHC? Qual o tópico? É o de gênero? (...)", recordando o tópico debatido na turma dele.

\section{Discussões, Implicações e Recomendações}

O Debate Estruturado deve coexistir com outras estratégias pedagógicas. Neste estudo, ele se mostrou robusto para ser aplicado no ensino de alguns conteúdos de interface e interação (privacidade, questões de gênero, aprendizado, robôs em redes sociais) em turmas de Engenharia de Software e de Sistemas de Informação. No entanto, ele não se aplica a todos os conteúdos trabalhados em IHC. Ele se soma às outras estratégias disponíveis. Por exemplo, nas turmas de 2018-I, outras práticas foram conduzidas em sala com os estudantes, como uma dinâmica de cardsorting e a avaliação heurística de um sistema interativo. Vídeos, demonstrações e trabalhos práticos de projeto e implementação também foram empregados.

Recomenda-se variar os tópicos e artigos de modo a permitir uma discussão diferente a cada debate e semestre. Eles podem ser alterados de modo a refletir os valores humanos que estão em evidência na comunidade, mantendo os debates atuais e envolventes. Isso ocorreu nas discussões sobre privacidade em Internet das Coisas e sobre a forma como a interface do Facebook pode ser opressora para pessoas com determinada identidade de gênero, $1^{\circ}$ e $2^{\circ}$ debates de 2017-II, respectivamente. A leitura dos artigos alinha as discussões em sala com as discussões mais recentes nas conferências e familiariza os estudantes com o estilo de escrita e métodos empregados nos artigos da área, potencializando o seu aprendizado.

Recomenda-se usar o debate como elemento integrador na formação de competências associadas aos valores humanos em IHC. O debate deve ser conduzido após os estudantes já terem familiaridade com alguns tópicos da disciplina. Essa é a razão de ocorrerem apenas 2 debates por semestre, sendo o primeiro com quase dois meses de aula e o segundo próximo ao término do semestre. O debate que ocorre próximo ao fim do semestre possibilita ao estudante articular e inter-relacionar os diversos conceitos, técnicas e métodos estudados na disciplina.

Recomenda-se empregar o debate de forma estruturada e focada. Pode-se questionar se o debate não deve ser mais livre para interação entre os estudantes e contendo diversos lados, em vez de apenas 2. Acredita-se que algum nível de estruturação é necessário para que as discussões ocorram de forma ordenada, com preparação prévia e para que o debate se mantenha focado. É fundamental que o debate tenha apenas um tópico chave e que seja norteado pela leitura de artigos.

Por fim, é importante destacar que as ideias da aprendizagem ativa e da aprendizagem colaborativa presentes no Debate Estruturado buscam permitir que o estudante adquira novos conhecimentos a partir de seus conhecimentos e experiências e que o motive a analisar as diferenças de opiniões e a tirar suas próprias conclusões a partir do diálogo com os colegas. Assumir uma posição e depois adaptá-la a outras percepções é fundamental ao se lidar com valores que são complexos. Isso é algo observado frequentemente ao longo do debate.

\section{CONCLUSÕES}

Buscam-se constantemente novas estratégias pedagógicas que engajem os estudantes e os auxiliem no desenvolvimento de competências e habilidades. Neste estudo, apresenta-se a estratégia de Debate Estruturado como uma alternativa no processo de ensino-aprendizagem de valores humanos em IHC. Descreve-se a estratégia e relata-se a experiência com 9 debates conduzidos, em 5 turmas de 2 cursos de graduação.

Experiências com estudos de caso do Debate Estruturado sugerem que ele é capaz de engajar estudantes de IHC. Ao perceber os diversos pontos de vista acerca de um fator humano no sistema, o estudante desenvolve a sensibilidade para o tópico, reconhecendo a existência de diferentes percepções e alternativas de projeto para lidar com ele. $\mathrm{O}$ debate também funciona como elemento aglutinador, levando o estudante a inter-relacionar diversos conceitos de IHC, como os fundamentos teóricos, projeto e avaliação de sistemas interativos. 
Entre as análises que permanecem para serem feitas em trabalhos futuros estão: número de debates mais adequado para se conduzir em um semestre de duração da disciplina, conteúdos da disciplina que possam ser adequados ao uso do debate como elemento aglutinador, nível de aprendizagem dos estudantes quando se usa a estratégia de Debate Estruturado. Esperamos que este trabalho motive novas discussões e estratégias pedagógicas para ensino de valores humanos em IHC.

\section{REFERÊNCIAS}

1. Robert Bogdan and Sari Knopp Biklen. 1992. Qualitative research for education. Allyn \& Bacon Boston.

2. Charles C Bonwell and James A Eison. 1991. Active Learning: Creating Excitement in the Classroom. 1991 ASHE-ERIC Higher Education Reports. ERIC.

3. Clodis Boscarioli, Luciana Zaina, Sílvia Bim, Simone Barbosa, and Milene Silveira. 2016. HCI Education in Brazil from the Results of the Workshop on Teaching of HCI. In 15th Brazilian Symposium on Human Factors in Computing Systems. ACM, USA, 52:1-52:4.

4. Elizabeth Churchill, Anne Bowser, and Jennifer Preece. 2013. Teaching and learning human-computer interaction: past, present, and future. Interactions 20, 2 (2013), 44-53.

5. Michele Darby. 2007. Debate: A teaching-learning strategy for developing competence in communication and critical thinking. J Dent Hyg 81, 4 (2007).

6. Eliane de Araujo, Matheus Gaudencio, Andrey Menezes, Iury Ferreira, Iara Ribeiro, Alberto Fagner, Lesandro Ponciano, Fábio Morais, Dalton Guerrero, and Jorge Figueiredo. O papel do hábito de estudo no desempenho do aluno de programação. In Workshop sobre Educação em Computação (WEI). 730-738.

7. Rafael Ferreira, Glívia Barbosa, Carolina Andrade, Ismael Silva, and Flávio Coutinho. 2017. Caracterização de Opressão de Gênero em Redes Sociais a partir de Violações dos Princípios de Afetibilidade: Um estudo de caso no Facebook. In XIV Simpósio Brasileiro de Sistemas Colaborativos. 1486-1500.

8. Diego Fontdevila. 2017. Tales from an Agile Journey: Designing Curricula for Millennials in Industry and Academia. In 1st International Workshop on Software Engineering Curricula for Millennials. IEEE, USA, 2-2.

9. Rodrigo Guimarães, Andrea Mattos, and Carlos Cardonha. 2017. A Comparative Study of Technology-Mediated Learning Strategies for Teaching People with Intellectual Disability. In XVI Brazilian Symposium on Human Factors in Computing Systems. ACM, USA, 72:1-72:4.

10. Thomas T Hewett, Ronald Baecker, Stuart Card, Tom Carey, Jean Gasen, Marilyn Mantei, Gary Perlman, Gary Strong, and William Verplank. 1992. ACM SIGCHI curricula for human-computer interaction. ACM.

11. Jean Scherz Huryn. 1986. Debating as a teaching technique. Teach Sociol 14, 4 (1986), 266-269.
12. Gary Katzenstein. 1996. The Debate on Structured Debate: Toward a Unified Theory. Organ Behav Hum Decis Process 66, 3 (1996), 316 - 332.

13. Katerina Kedraka and Yiannis Kourkoutas. 2018. Debates in Teaching Bioethics. J Curric Teach 7, 1 (2018), 32.

14. Marc Langheinrich. 2001. Privacy by Design - Principles of Privacy-Aware Ubiquitous Systems. In 3rd International Conference on Ubiquitous Computing. Springer-Verlag, Berlin, 273-291.

15. Carla Leitão, Cristiano Maciel, Lara Piccolo, Luciana Salgado, Patricia de Souza, Raquel Prates, Roberto Pereira, and Vinicius Pereira. 2017. Human Values in HCI: A Challenge for the GrandIHC-BR. In XVI Brazilian Symposium on Human Factors in Computing Systems. ACM, USA, 70:1-70:6.

16. D Scott McCrickard, Christa M Chewar, and Jacob Somervell. 2004. Design, science, and engineering topics?: teaching HCI with a unified method. In ACM SIGCSE Bulletin, Vol. 36. ACM, 31-35.

17. Talita Menezes, Joanna Marques, and Nazareno Andrade. 2017. Me sinto de mãos dadas! Um estudo sobre efeitos de comunidade no comportamento online de suas participantes. In XIV Simpósio Brasileiro de Sistemas Colaborativos. 1414-1428.

18. Maria Augusta Vieira Nelson, Rommel Vieira Carneiro, and Marco Rodrigo Costa. 2017. Interdisciplinary Software Projects As an Active Methodology to Practice for the Profession. In 1st International Workshop on Software Engineering Curricula for Millennials. IEEE Press, USA, 28-32.

19. Lesandro Ponciano and Nazareno Andrade. 2018. Perspectivas em Computação Social. In Computação Brasil, Raquel Prates and Thais Castro (Eds.). Vol. 36. Sociedade Brasileira de Computação, 30-33.

20. Lesandro Ponciano, Pedro Barbosa, Francisco Brasileiro, Andrey Brito, and Nazareno Andrade. 2017. Designing for Pragmatists and Fundamentalists: Privacy Concerns and Attitudes on the Internet of Things. In XVI Brazilian Symposium on Human Factors in Computing Systems. ACM, USA, 21:1-21:10.

21. Michael Prince. 2004. Does active learning work? A review of the research. Journal of Engineering Education 93, 3 (2004), 223-231.

22. Marco Ruediger, Amaro Grassi, Ana Freitas, Andressa Contarato, Carolina Taboada, Danilo Carvalho, Ferreira, and others. 2017. Robôs, redes sociais e política no Brasil: estudo sobre interferências ilegítimas no debate público na web, riscos à democracia e processo eleitoral de 2018. FGV DAPP.

23. Andreza Valgas, Felipe Carmo, Lesandro Ponciano, and Luís Góes. 2017. Análise da Popularidade, Visibilidade e Atividade de Diferentes Tipos de Robôs na Rede Social Twitter. In XIV Simpósio Brasileiro de Sistemas Colaborativos. 1342-1356. 